\title{
Examination of Trichoderma phylogenies derived from ribosomal DNA sequence data
}

\author{
SARAH L. DODD ${ }^{1 *}$, ROSS N. CROWHURST ${ }^{2}$, ALLEN G. RODRIGO ${ }^{3}$, GARY J. SAMUELS ${ }^{4}$, \\ ROBERT A. HILL ${ }^{5}$ AND ALISON STEWART 6 \\ ${ }^{1}$ School of Biological Sciences, University of Auckland, Private Bag 92 017, Auckland, New Zealand \\ ${ }^{2}$ Horticulture and Food Research Institute of NZ, Mt Albert Research Centre, Private Bag 92 169, Auckland, New Zealand \\ ${ }^{3}$ Department of Microbiology, University of Washington, Box 357740, Seattle, WA 98195-7740, U.S.A. \\ ${ }^{4}$ United States Department of Agriculture, Agriculture Research Service, Systematic Botany and Mycology Laboratory, Room 304, B-011A. \\ BARC-W, Beltsville, MD 20705-2350, U.S.A. \\ ${ }^{5}$ Horticulture and Food Research Institute of NZ, Ruakura Research Station, Private Bag 3123, Hamilton, New Zealand \\ ${ }^{6}$ Soil Plant and Ecological Sciences Division, Lincoln University, P.O. Box 84, Canterbury, New Zealand
}

\begin{abstract}
Ribosomal DNA sequences were assessed for their usefulness in distinguishing among Trichoderma isolates and for their robustness in resolving their phylogenetic relationships. DNA sequences from the D2 region of the 28S rRNA gene were determined for 50 Trichoderma isolates representing seven species. Eight distinct sequence types existed, and were mostly consistent with groupings based on morphology. Sequence variability within the D2 region alone was not sufficient to provide a reliable phylogeny. Sequences from the ITS1, $5.8 \mathrm{~S}$ and ITS2 regions were subsequently determined for 18 of the isolates. Eight distinct ITS sequence types were detected among these 18 isolates. The ITS sequence types were generally consistent with morphology, ITS1 sequence data supported the identification of the Th3 T. harzianum group of Muthumeenakshi et al. (1994) as T. atroviride. The data also confirmed that the biocontrol strains of this study were different from those causing disease problems in the mushroom industry in Europe and North America. Results from the phylogenetic analysis stress the importance of testing the robustness of data used to predict phylogenies. Two ITS sequence data sets for the same group of isolates produced significantly different phylogenies. Congruence analysis detected that T. inhamatum (GJS90-90) was corrupting tree topologies and 'first order pruning' was performed by removing its sequence from the two ITS data sets. Subsequent differences in the topologies of pruned ITS1 and ITS2 trees were attributed to a lack of phylogenetic information in the ITS2 sequence region. Although ITS sequences successfully differentiated among morphologically distinct isolates within Trichoderma, it did not provide a sufficient phylogenetic signal to resolve all of their relationships.
\end{abstract}

Trichoderma consists of a number of economically important strains, valued for their biocontrol capabilities and ability to produce beneficial metabolites and enzymes (Papavizas, 1985; Ghisalberti \& Sivasithamparam, 1991; Buchert et al., 1992; Schirmbock et al., 1994). In contrast to their usefulness to industry, however, there have also been reports of pathogenic strains that pose a threat to both horticulture and human health (Loeppke et al., 1983; Seaby, 1987; Menzies, 1993).

Differentiation of such isolates within Trichoderma has proved difficult due to the limited number of easily identifiable cultural and morphological characters available for comparison (Rifai, 1969; Bissett, 1984, 1991a-c, 1992). Consequently, the taxonomy of the genus is currently under review with a variety of morphological, cultural, molecular and biochemical characteristics being used to define isolates (see review by Samuels, 1996).

* Current address: Soil Plant and Ecological Sciences Division, Lincoln University, P.O. Box 84, Canterbury, New Zealand.
For this study, a method was sought that would differentiate Trichoderma isolates exhibiting good biological control activity against plant pathogenic Armillaria species. In particular, sequence data from the ribosomal gene cluster was investigated because of the successful use of such data in distinguishing among Trichoderma isolates (Muthumeenakshi et al., 1994; Ospina-Giraldo et al., 1998). In addition, sequence data may be used to reconstruct the phylogeny of a group of isolates, thus providing valuable insights into their evolutionary relationships. Thus, in turn, may lead to a more efficient selection and use of such isolates for commercial applications. We also wished to use the sequence data to determine if our biocontrol agents were identical to those identified as a problem in the mushroom industry in Europe and North America (Muthumeenakshi et al., 1994; Muthumeenakshi, Brown \& Mills, 1998; Ospina-Giraldo et al., 1998).

Previous studies have used sequences from the ribosomal gene cluster to distinguish among, and predict phylogenies 
Table 1. Origin, morphological and molecular characterisation of Trichoderma isolates

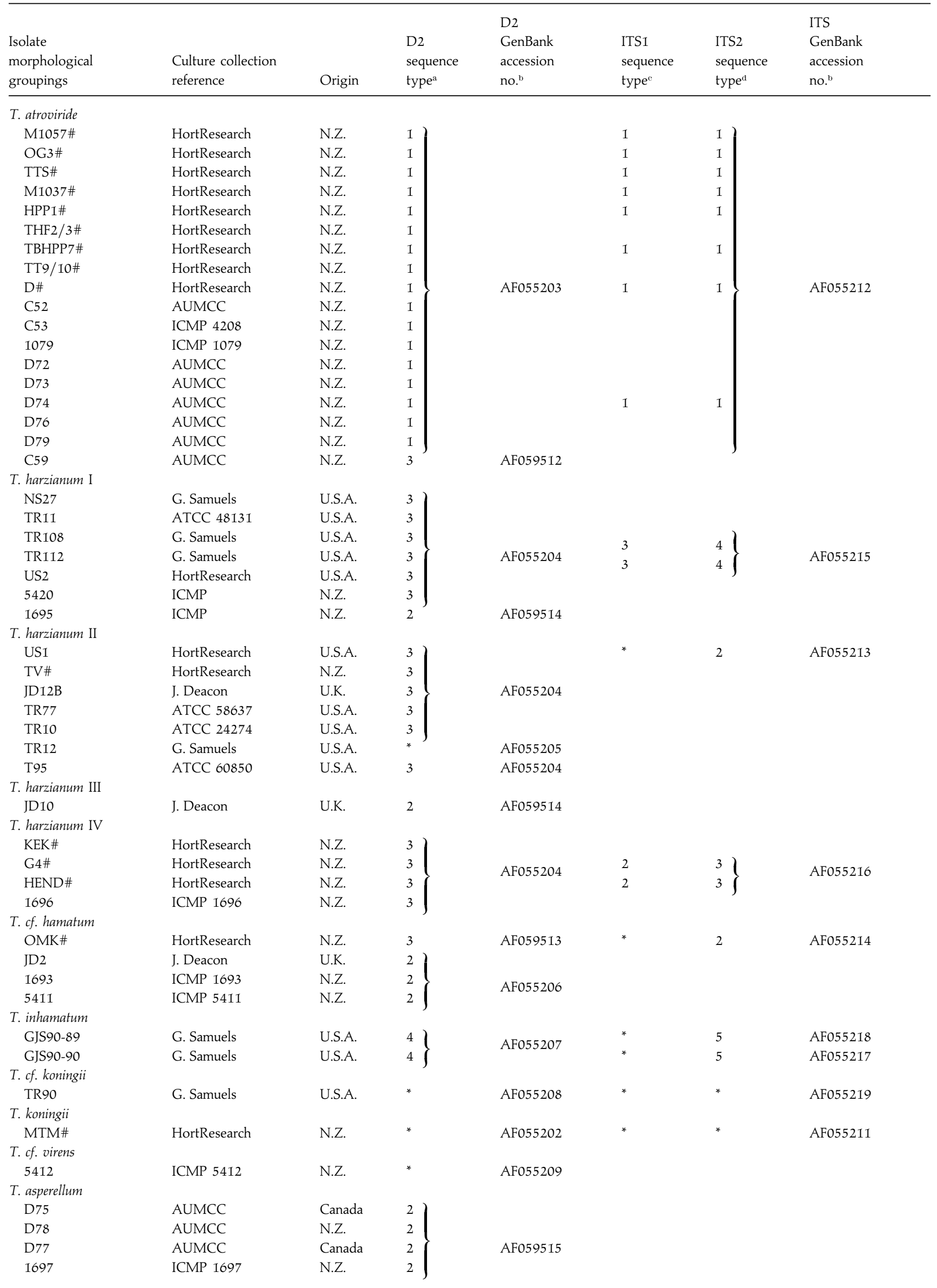


for, isolates of Trichoderma (Muthumeenakshi et al., 1994; Rehner \& Samuels, 1994; Schlick et al., 1994; Kuhls et al., 1997). There has, however, been no detailed analysis of the adequacy of the data or the robustness of the reconstructed phylogenies. In the present study, we assessed the level of variability and determined the phylogenetic robustness of sequence data from the D2 region of the 28S rRNA gene for 50 Trichoderma isolates, including 15 from New Zealand with biocontrol activity. Similarly, sequences from the ITS1, ITS2 and combined ITS1, 5.8S and ITS2 regions were assessed for 18 of these isolates. Our results indicate that ITS sequences may be used to reconstruct the evolutionary relationships amongst isolates of Trichoderma. These evolutionary relationships appear to agree closely with those obtained using morphological information. Nonetheless, phylogenies of ITS1 and ITS2 may be incongruent, although much of this may be attributed to the absence of sufficient phylogenetic signal in these regions.

\section{MATERIALS AND METHODS}

\section{Fungal cultures}

Morphological identity, source, geographical origin, and sequence type of the 50 Trichoderma isolates investigated are listed in Table 1. All isolates were identified morphologically to species and sub-species level. T. harzianum Rifai isolates were further differentiated into four subgroups I-IV based on morphological characters.

\section{Amplification of rDNA}

The D2 region of the $28 \mathrm{~S}$ rDNA molecule was amplified and sequenced for all 50 Trichoderma isolates (Table 1 ) as well as for a single representative isolate of Fusarium oxysporum (FO). Similarly, the ITS region was amplified and sequenced for 18 of the 50 Trichoderma isolates (Table 1), as well as isolate FO. DNA was extracted from cultures using the method described by Crowhurst et al. (1991) and further purified with the Wizard $^{\text {(m) }}$ DNA Clean-Up System (Promega Corporation, Madison, WI, U.S.A.). The D2 region was amplified using the primers P2 (GAAAAGAACTTTGAAAAGAGAGTG) and P3 (CCTTGGTCCGTGTTTCAAGACG), which were based on those described in Guadet et al. (1989). Amplification of the ITS1 and ITS2 regions, as well as the $5.8 \mathrm{~S}$ rDNA region was performed using primers ITS4 and ITS5 (White et al., 1990).
Amplification reactions were performed in a volume of $100 \mu \mathrm{l}$ containing $10 \mathrm{~mm}$ Tris- $\mathrm{HCl} \mathrm{pH} 8.3,50 \mathrm{~mm} \mathrm{KCl}, \quad 1.5 \mathrm{~mm}$ $\mathrm{MgCl}_{2}, 100 \mathrm{~mm}$ each of dATP, dCTP, dGTP and dTTP (Boehringer Mannheim, Mannheim, Germany), 20 pmol each of the two appropriate primers, 40-100 ng of genomic DNA and $0.5 \mathrm{U}$ of Taq DNA polymerase (Life Technologies Inc., Gaithersburg, MD, U.S.A.). Amplification was carried out in an Ericomp Twinblock Easy Cycler programmed for 3 min at $94{ }^{\circ} \mathrm{C}$ followed by 30 cycles of $30 \mathrm{~s}$ at $94^{\circ}, 30 \mathrm{~s}$ at $55^{\circ}$ and $30 \mathrm{~s}$ at $72^{\circ}$, followed by a final $7 \mathrm{~min}$ at $72^{\circ}$. Two replicate reactions were performed per isolate. Following amplification, the two reactions were pooled and purified using a Wizard ${ }^{\boxplus}$ PCR Clean-Up System (Promega). Purified products were subject to direct sequencing by the Centre for Gene Research, Department of Biochemistry, University of Otago, Dunedin, N.Z. All products were sequenced in both forward and reverse orientations.

\section{Alignment of sequence data and cluster analysis}

For each sequence region analysed (D2, ITS1, ITS2 or combined ITS1, 5.8S and ITS2 regions), multiple sequences were aligned using the computer program PILEUP (Wisconsin Sequence Analysis Package, Version 8, Genetics Computer Group Inc., Madison, WI, U.S.A.) and options GapWeight $=2 \cdot 0$ and LengthGapWeight $=0 \cdot 3$, A UPGMA dendrogram was produced for each data set and isolate groupings were assessed visually. Isolates with identical sequences in a particular rDNA region were removed so that each sequence type was represented once only in subsequent analyses.

\section{Outgroup selection}

Using the computer program MEGA 1.01 (Kumar, Tamura \& Nei, 1993), a preliminary neighbour-joining tree (NJ) (Saitou \& Nei, 1987) was constructed for the four data sets (D2, ITS1, ITS2 and combined ITS1, 5.8S and ITS2). The Jukes-Cantor distance model (Jukes \& Cantor, 1969) was employed and F. oxysporum FO (GenBank accession no. AF055220) used as the outgroup. FO was tested as an outgroup for all data sets in order to assess the level of reliability in using an isolate from the same order (i.e. Hypocreales) to root derived trees. Unrooted topologies were constructed using the five taxa (M1057, MTM, US1, TR90 and GJS90-90) common to all four data sets. Alignment gaps (i.e. putative insertion/deletion events, or indels) were deleted using the 'pairwise-deletion'

All isolates identified by G.J.S. T. harzianum isolates were differentiated further into subgroups I-IV based on morphological characters. Subgroup (I) consisting of a miscellaneous group of isolates with general $T$. harzianum morphologies. (II) No odour; subglobose conidia $<3 \mu \mathrm{m}$ diam., $\mathrm{L} / \mathrm{W}=1 \cdot 1-1 \cdot 2 \mu \mathrm{m}$; phialides squat, $7 \mu \mathrm{m}$ long, L/mid point width $=<2 \cdot 4 \mu \mathrm{m}$. (III) No odour; large warted conidia, $3 \cdot 8 \mu \mathrm{m}$ average diam., $\mathrm{L} / \mathrm{W} 1 \cdot 2 \mu \mathrm{m}$; long phialides $8 \mu \mathrm{m}$, $\mathrm{L} /$ mid point width $2.5 \mu \mathrm{m}$. (IV) No odour; yellow pigment on PDA, conidia $<3 \mu \mathrm{m}$ diam., L/W $1 \cdot 1-1 \cdot 3 \mu \mathrm{m}$; phialides variable but $<8 \mu \mathrm{m}$ long, L/mid point width $=2-2.5 \mu \mathrm{m}$. Based on morphology, T. cf. hamatum isolates belong to the complex that Rifai (1969) referred to as the T. hamatum species-aggregate.

(\#) Isolates with biocontrol activity against Armillaria.

a, c, d Isolates with the same number had identical sequence. $\left(^{*}\right)$ indicates isolates that possessed unique sequence. Isolates with no numbers were not tested.

b For a particular species, each unique sequence type is represented only once in GenBank.

HortResearch, N.Z. - collection of R. Hill, Horticultural and Food Research Institute of New Zealand Ltd, Rurakura Research Centre, Private Bag 3123, Hamilton, N.Z.; ICMP - International Collection of Microorganisms, Landcare Research Ltd, Mt Albert Research Centre, Private Bag, 92 169, Auckland, N.Z.; AUMCC - Auckland University Microbial Culture Collection, School of Biological Sciences, University of Auckland, Private Bag 92019, Auckland, N.Z.; J. Deacon - collection of J. Deacon, Institute of Cell and Molecular Biology, University of Edinburgh, Edinburgh, EH9 3JH, U.K.: G. Samuels - collection of G. J. Samuels, USDA-ARS, Systematic Botany and Mycology Laboratory, Beltsville, Maryland 20705, U.S.A. 
option in MEGA. An indication of the level of confidence given for interior branches was achieved by generating 1000 bootstrap pseudoreplicates (Felsenstein, 1985). The rooted and unrooted topologies constructed using different data sets were compared visually.

To find suitable outgroup sequences for the ITS data sets, a Blast search (Altschul et al., 1990) was performed using ITS1, $5.8 \mathrm{~S}$ and ITS2 sequence of the T. atroviride Karst. isolate, M1057. Phylogenetic signal was then determined for 14 sequences identified as most similar to M1057 by calculating the mean number of transition substitutions between each of these sequences and every ingroup sequence (Hillis, Allard \& Miyamoto, 1993).

To determine whether the use of four outgroups would be more reliable than one, topologies were compared for a second set of preliminary $\mathrm{NJ}$ trees, constructed for each of the three ITS data sets. In this instance, Neotyphodium uncinatum (GenBank L20305), Neotyphodium sp. (L07134), Epichloë sp. (L07131) and F. oxysporum (AF055220) were specified as the outgroups. FO was included for consistency among data sets.

Neighbour-joining analysis. NJ trees were constructed for each of the four data sets (D2, ITS1, ITS2 or combined ITS1, $5.8 \mathrm{~S}$ and ITS2 sequence) using the Kimura-2-parameter distance model (Kimura, 1980) and the pairwise deletion of gaps option. For the tree constructed from the D2 sequence data, $\mathrm{FO}$ was specified as the outgroup. For trees constructed from the three ITS sequence data sets, isolate FO plus $N$. uncinatum, Neotyphodium sp., Epichloë sp. (see above) were specified as the outgroups. One thousand bootstrap replications were performed to assess the level of support for each node. Nodes with bootstrap support of $<80 \%$ were considered to be not significantly supported.

Parsimony analysis. Most parsimonious (MP) estimates of the phylogenetic tree were generated for the D2 and the three ITS sequence data sets using PAUP 3.1.I (Swofford, 1993). Twenty initial trees were generated using random sequence addition. Branch swapping was performed using the treebisection-reconnection algorithm. One thousand bootstrap replicates were also generated using these options. Gaps were treated as missing data. Outgroups were specified for each data set as described in the NJ analysis. Where four outgroups were employed, the ingroup was specified as monophyletic and the outgroup paraphyletic. A 50\% majority rule consensus tree (Margush \& McMorris, 1981) was calculated from the equally parsimonious trees generated for each data set. Consistency index (CI) (Kluge \& Farris, 1969) was calculated for each consensus tree to give an indication of the robustness of tree topologies.

\section{Congruence studies of ITS1 and ITS2 sequence data}

Comparison of tree topologies. Initially, both rooted and unrooted topologies of all the phylogenetic trees were compared to determine if the different data sets and different tree building methods produced the same phylogeny. Unrooted trees for all phylogenetic trees were constructed using the isolates present in all four data sets (M1057, MTM, GJS90-90, TR90 and US1), excluding the outgroups.

Following this, a test based on that of Rodrigo et al. (1993) was performed to identify if differences observed in the topologies of the phylogenetic trees generated from the ITSI and ITS2 data sets were significantly different or the result of sampling error. MP trees were generated from both the ITSI and ITS2 sequence data sets using the seven taxa common to both data sets (MTM, M1057, GJS90-90, TR112, US1, HEND and TR90), as well as the four outgroups mentioned previously. These trees were generated using the heuristic search method within PAUP 3.1.1 and using 20 bootstrap test replications. Equally parsimonious trees generated from the two data sets were then compared to determine if the two data sets shared trees in common. Zero tree to tree distance values were used to determine trees common to both data sets.

First-order pruning. This is a procedure where the sequence data for a single taxon are removed from both data sets to remove possible misinformative data (Rodrigo et al., 1993). This procedure was performed by first generating a 50\% majority rule consensus tree, from the equally parsimonious trees generated in the above topology analysis, for each of the ITS sequence data sets. ITS1 and ITS2 consensus trees were then compared to determine if the placement of one (or a few) taxon was responsible for the differences observed between the tree topologies. Sequence for GJS90-90 was subsequently removed from each of the two ITS data sets and a second bootstrapped heuristic search performed on each of the pruned data sets. Trees generated for the two data sets were again compared to determine if they shared topologies in common using zero tree to tree distance values. Average, maximum and minimum tree to tree distance values were also calculated.

A comparison was then undertaken to determine whether the differences observed between trees of the two pruned data sets fell within the range of natural variation observed among trees of a single data set. Initially, the level of variation existing between trees of the two data sets was calculated. A non-bootstrapped heuristic search was subsequently performed on each of the pruned ITS1 and ITS2 data sets separately, using TBR branch swapping and 20 replications of random taxon addition options within PAUP 3.1.1. Pruned ITS1 trees were then compared to pruned ITS2 trees using tree to tree distance values. Average, maximum and minimum distance values were also calculated.

Secondly, the level of variation within each data set was determined. Pairwise distances were calculated between trees within each of the two ITS data sets. For each data set, a maximum tree to tree distance value was calculated, as well as the percentage of the tree to tree distances that were greater than the average tree to tree distance observed between the two data sets.

Finally, the number of steps (i.e. increase in tree length) required to produce the trees of one data set from the sequence data of the other, was calculated. For this step, one of the pruned ITS sequence data sets was 'executed' under PAUP 3.1.1 while the trees generated from the heuristic search of the other pruned ITS data set, were loaded using the 
'load constraints' option. The new tree length was then determined using the 'describe trees' option, and the difference in tree length between these trees and those of the data set whose sequence data was 'executed' was calculated. This calculation was performed for both ITS data sets.

\section{RESULTS}

\section{Sequence data}

Eight distinct sequence types were found in the D2 region of the $28 \mathrm{~S}$ rDNA molecule for 50 Trichoderma isolates (Table 1). Eighteen representative isolates were selected to determine whether sequences from the ITS region gave greater differentiation between isolates than D2 sequences. Representatives were mainly from the two biggest D2 sequence groups (Groups 1 and 3), but also included the two $T$. inhamatum Veerkamp \& W. Gams isolates and the five with unique D2 sequences. Isolates were selected to provide the greatest information for a small sample size. Eight distinct ITS1 and seven distinct ITS2 sequence types were determined for the 18 isolates (Table 1 ). In contrast, $5.8 \mathrm{~S}$ rDNA sequence was totally conserved among these 18 Trichoderma isolates but was used to assist in the alignment of non-homologous ITS regions among the 18 isolates.

ITS1 sequence data was the most variable of the three regions investigated, followed by ITS2 and then the D2 regions (Table 2). All but ITSI sequences appeared to have a GC base bias according to the $x^{2}$ test $(P=0.01)$. The ITS2 sequence showed the greatest bias with $65 \%$ GC content, followed by D2 (61\%) and finally the combined ITS and $5.8 \mathrm{~S}$ region $(56 \%)$.

\section{Cluster analysis}

D2 sequence. Cluster analysis of sequence data from the D2 region revealed four main sequence types and four unique sequences among the 50 isolates. D2 sequence types were generally consistent with morphologically based groupings but not with isolate origin since the origin of isolates with Type 2 and 3 sequence overlapped (Table 1)

ITS sequence. When the ITS sequence of isolates with identical D2 sequence was analysed, isolates with Type 1, 3 and 4 D2 sequence all formed their own discrete clusters within each of the three ITS data sets (i.e. data sets consisting of ITS1, ITS2 or combined ITS1, 5.8S and ITS2 sequence). None of the ITS sequence data sets further differentiated the T. atroviride isolates with Type 1 D2 sequence. In contrast, Type 3 D2 isolates were further differentiated with ITS1 and, for the most part, ITS2 sequence and their groupings were consistent with morphology.

Table 3. Mean transition frequencies for pairwise comparisons between sequence from each outgroup test organism and sequences of the 18 Trichoderma isolates in each of the three ITS sequence data sets

\begin{tabular}{lllll}
\hline & & \multicolumn{2}{l}{ Transition frequencies } \\
\cline { 4 - 5 } & GenBank & & \\
& accession no. & ITS1 & ITS2 & ITS1, 5.8S + ITS2 \\
\hline Cladosporium sphaerospermum Penz. & L25433 & $0 \cdot 47$ & $0 \cdot 27$ & $0 \cdot 40$ \\
Neotyphodium uncinatum (W. Gams, Petrini \& D. & L20305 & $0 \cdot 45$ & $0 \cdot 48$ & $0 \cdot 47$ \\
Schmidt) Glenn, Bacon \& Hamlin & & & & \\
N. uncinatum & L07135 & $0 \cdot 42$ & $0 \cdot 49$ & $0 \cdot 42$ \\
Neotyphodium sp. & L07134 & $0 \cdot 41$ & $0 \cdot 49$ & $0 \cdot 42$ \\
Fusarium oxysporum Schltdl. & AF055220 & $0 \cdot 38$ & $0 \cdot 37$ & $0 \cdot 41$ \\
N. uncinatum & L07128 & $0 \cdot 37$ & $0 \cdot 48$ & $0 \cdot 46$ \\
Epichloë sp. & L07131 & $0 \cdot 37$ & $0 \cdot 43$ & $0 \cdot 39$ \\
Fusarium sambucinum Fuckel & X65480 & $0 \cdot 34$ & $0 \cdot 47$ & $0 \cdot 45$ \\
E. typhina (Pers.) Tul & L07133 & $0 \cdot 29$ & $0 \cdot 48$ & $0 \cdot 38$ \\
Gaeumannomyces graminis (Sacc.) Arx \& D. L. Olivier & U17207 & $0 \cdot 27$ & $0 \cdot 41$ & $0 \cdot 41$ \\
E. typhina & L07132 & $0 \cdot 25$ & $0 \cdot 48$ & $0 \cdot 38$ \\
E. amarillans J. F. White & L07129 & $0 \cdot 25$ & $0 \cdot 45$ & $0 \cdot 37$ \\
Magnaporthe grisea (T. T. Hebert) M. E. Barr & U17329 & $0 \cdot 25$ & $0 \cdot 44$ & $0 \cdot 41$ \\
Phialophora graminicola (Deacon) J. Walker & U17217 & $0 \cdot 24$ & $0 \cdot 35$ & $0 \cdot 31$ \\
Acremonium lolii Latch, M.J.C. \& Samuels & L07130 & $0 \cdot 23$ & $0 \cdot 47$ & $0 \cdot 35$ \\
\hline
\end{tabular}

* Sequence alignments available from Sarah Dodd, Soil Plant and Ecological Sciences Division, Lincoln University, N.Z.

Table 2. Properties of Trichoderma sequence data*

\begin{tabular}{llllll}
\hline & $\begin{array}{l}\text { Length of sequence } \\
\text { (bp) }\end{array}$ & $\begin{array}{l}\text { Sequence types } \\
\text { shared by }>\text { i isolate } \\
(\%)\end{array}$ & $\begin{array}{l}\text { Variable sites } \\
(\%)\end{array}$ & $\begin{array}{l}\text { Alignment gaps } \\
(\text { no. })\end{array}$ & $\begin{array}{l}\text { G }+C \\
(\%)\end{array}$ \\
\hline Sequence region & 199 & 50 & 11 & 6 & 61 \\
ITS1 & 213 & 50 & 24 & 88 & 53 \\
ITS2 & 180 & 71 & 18 & 44 & 65 \\
ITS1, 5.8S and ITS2 & 557 & 50 & 16 & 132 & 56 \\
\hline
\end{tabular}

* Isolate whose ITS sequence was obtained as described in Materials and Methods. 
(a)

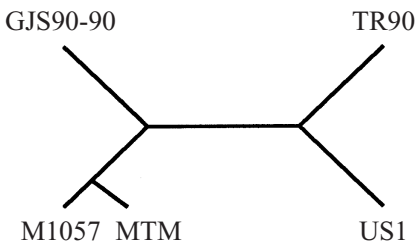

(b)

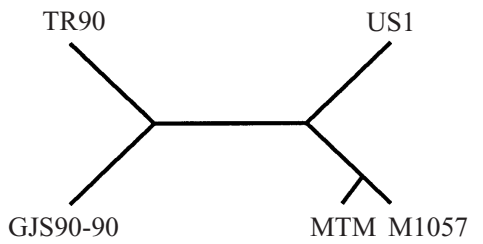

Fig. 1. The two unrooted topologies derived from all phylogenetic trees using the taxa common to all data sets, excluding the outgroup Fusarium oxysporum. Tree (a) represents the unrooted topology of all D2, ITS1 and ITS2 neighbour-joining trees and the neighbourjoining tree of the combined ITS sequence data set where $F$. oxysporum was specified as the outgroup. It also represents the unrooted topology of ITS1 and ITS2 parsimony trees. Tree $(b)$ represents the unrooted topology of the neighbour-joining tree of the combined ITS sequence data set where four outgroups were specified.

The two T. inhamatum isolates (GJS90-89 and GJS90-90) with Type 4 D2 sequence differed from one another by only one nucleotide in the ITSI region. The three isolates with unique D2 sequence (MTM, TR90 and FO) also possessed unique sequence in both ITS regions.

\section{Outgroup selection}

To ensure the correct placement of an outgroup within a tree, the outgroup sequence must contain sufficient comparable phylogenetic signal to the ingroup sequences. The level of phylogenetic signal is considered sufficient for analysis if the mean number of transition substitutions between the outgroup sequence and each of the ingroup sequences is greater than $50 \%$ (Hillis et al., 1993). A mean transition frequency of $54 \%$ existed between D2 sequence data from the F. oxysporum outgroup and that of the Trichoderma ingroup. It was, therefore, assumed that sufficient phylogenetic signal existed between these sequences. In contrast, sequence of $F$. oxysporum produced mean transition frequencies of less than $50 \%$ with all three ITS sequence data sets (Table 3 ) indicating insufficient signal for rooting. Lack of signal was also indicated by the fact that differences were observed among the topologies of the four preliminary NJ trees (not shown), yet all four data sets produced the same unrooted topology (Fig. Ia).

The 14 sequences selected from the GenBank database search, and showing the greatest sequence similarity to the ITS sequence of M1057, were found to all have mean transition frequencies of less than $50 \%$ with the 18 corresponding Trichoderma sequences (Table 3). When the three fungi that gave the best overall transition frequencies for all three ITS data sets (Neotyphodium uncinatum, Neotyphodium sp. and Epichloë sp.) plus F. oxysporum were used as an outgroup, they were all placed in the same position for each of the three ITS trees (not shown). This result indicated an acceptable level of reliability.

\section{Neighbour-joining analysis}

D2 sequence. D2 sequence data produced a NJ tree in which only two clades were resolved (Fig. $2 a$ ), as indicated by a greater than $80 \%$ bootstrap support. These were the clade consisting of T. atroviride M1057 and T. koningii Oudem. MTM, and the clade with T. cf. virens (J. H. Mill., Giddens \& A. A. Foster) Arx 5412. Phylogenetic relationships among all other groups were not resolved.

ITS sequence. Sequence from the ITSI region provided the greatest amount of phylogenetic information out of the three regions investigated. The ITS1 tree was able to resolve five clades (Fig. 2b), whereas the ITS2 (Fig. $2 c$ ) and combined ITS sequence trees (Fig. $2 d$ ) could only resolve three.

Like the D2 NJ tree, all three ITS data sets gave high bootstrap support for the clade consisting of $T$. atroviride M1057 and T. koningii MTM. In addition, the three ITS trees all gave high bootstrap support for the clade consisting of isolates with Type 3 D2 sequence (Table 1, Fig. 2), T. harzianum I (TRI12), T. harzianum II (USI) and T. harzianum IV (HEND). T. cf. hamatum (Bonord.) Bainier OMK was also included in this clade for the ITSI and combined data sets. Although ITSI sequence was unable to further resolve the members of this clade, ITS2 sequence and combined ITS was able to distinguish the T. harzianum IV isolate HEND from the other members of the clade.

The ITSI tree also distinguished T. inhamatum (GJS90-89 and GJS90-90 from one another and T. cf. koningii TR90 from all other isolates. Similarly, the combined ITS tree was able to distinguish the T. inhamatum group.

Comparison of the topologies of the three ITS NJ trees revealed differences in the placement of taxa within the trees. The placement of taxa within ITS2 NJ tree appeared to be in the reverse order to that produced in the ITSI and combined ITS NJ trees. Only two of the main clades were, however, resolved within the ITS2 tree as opposed to four in the ITS1 tree, thus giving less support for the order of taxa within the ITS2 tree.

\section{Parsimony analysis}

The MP trees essentially produced the same isolate groupings and order of clades as the NJ trees for the corresponding data sets (Fig. 3). All four trees had high consistency indices indicating a high level of support for their topologies. Fewer groups were, however, resolved within each of the trees compared to the NJ method, as indicated by fewer clades with significant bootstrap support ( $>80 \%$ ). As with NJ trees, all four MP trees gave high bootstrap support fo the T. atroviride (M1057) and T. koningii (MTM) clade. Similarly, the MP D2 tree distinguished T. cf. virens 5412 from all other isolates, the 
(a) D2 28S NJ
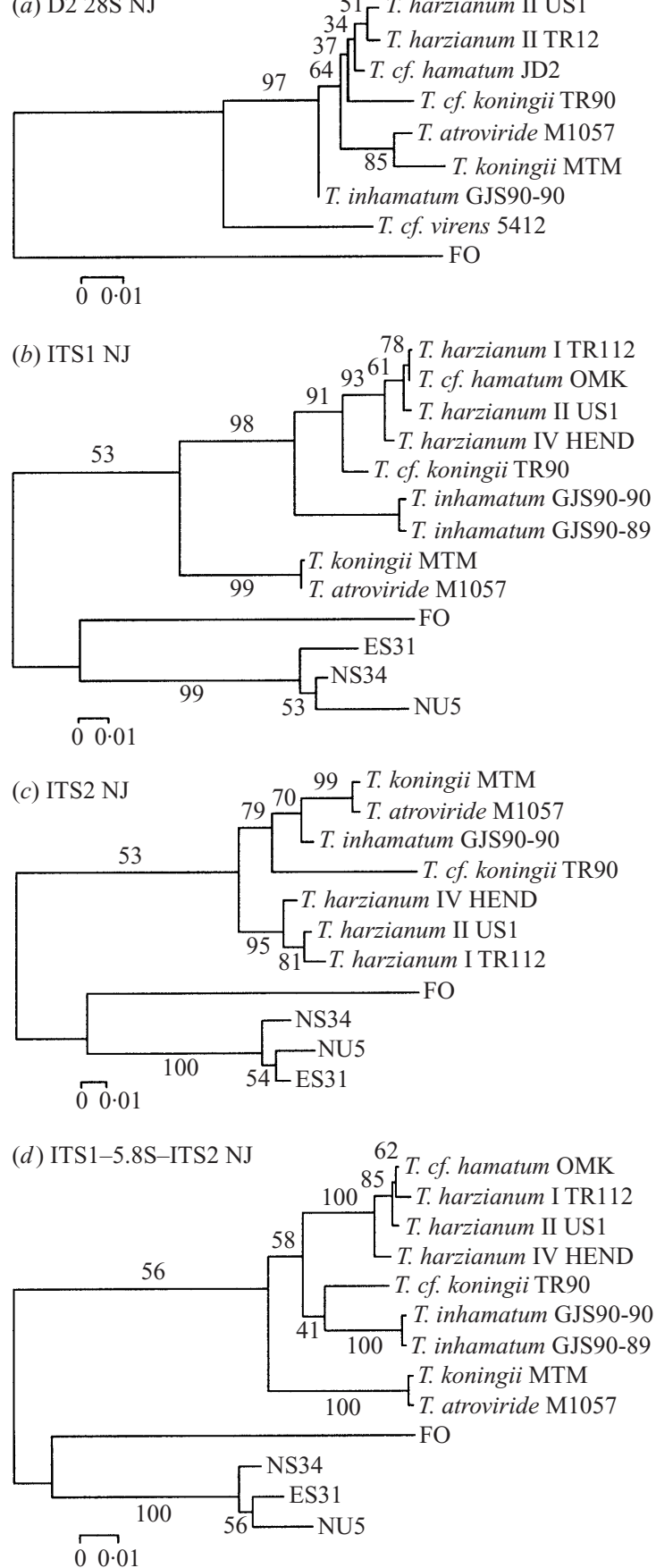

Fig. 2. Neighbour-joining (NJ) estimates of Trichoderma isolate phylogeny for sequence data from the (a) D2 region of the $28 \mathrm{~S}$ ribosomal gene, (b) ITS1 region, (c) ITS2 region and (d) ITS1, 5.8S and ITS2 combined regions of the ribosomal genes. Trees were generated using the Kimura-2-parameter distance model with Fusarium oxysporum (FO) specified as the outgroup for the D2 sequence tree. Neotyphodium uncinatum (NU5), Neotyphodium sp. (NS34), Epichloë sp. (ES31) and F. oxysporum (FO) were specified as the outgroups for the ITS sequence trees. Values associated with branches indicate the degree of bootstrap support expressed as percentage of 1000 bootstrapped trees in which the corresponding clades are present. Scale bars indicate horizontal branch length, expressed as the hypothesised number of nucleotide substitutions per site.

ITS1 tree distinguished the T. inhamatum GJS90-89 and GJS90-90 from other isolates and the ITS2 tree supported the clade of isolates (US1, TR112 and HEND) with Group 3 type
D2 sequence (Table 1, Fig. 3). Apart from these examples, no other groups were resolved within the four MP trees.

\section{Congruence studies for ITS1 and ITS2 sequence data}

Comparison of the unrooted topologies of each of the $\mathrm{NJ}$ and MP phylogenetic trees revealed two different topologies for the five taxa in common (Fig. 1). This suggests that the differences in topology were attributed to something other than the unreliable placement of outgroups. Note, unrooted trees could not be constructed from the D2 and combined ITS parsimony trees because two (US1 and TR90) of the five taxa were unresolved within these trees.

Comparison of tree topologies. No zero tree to tree distance values were generated when the equally parsimonious ITSI trees were compared to those of the ITS2 data set (i.e. no trees were found that were common to both data sets). Differences observed in the topologies were, therefore, significant and not due to sampling error.

First-order pruning. Inspection of the ITS1 and ITS2 consensus parsimony trees (Fig. 4) revealed the placement of taxon GJS90-90 was corrupting their topologies. Sequence data for GJS90-90 was subsequently pruned from both data sets and each set reanalysed (Fig. 5). Comparison of pruned ITS1 and ITS2 trees revealed $3 \cdot 4 \%$ of the total trees were common to both data sets. The average distance (i.e. measure of topological difference) between pruned ITS1 and ITS2 trees was 3.8 $(\operatorname{Max}=8, \min =2)$. When the distances among trees generated from the ITS1 data set were calculated, $33 \cdot 7 \%$ of the comparisons were found to differ by a distance greater than 3.8 (Max distance $=8$ ). Similarly, $29 \cdot 7 \%$ of the ITS2 tree comparisons were found to differ by a distance greater than $3.8(\mathrm{Max}=6)$. Since the average distance between pruned ITS1 and ITS2 trees fell within the range of the natural variation observed within each data set, and the maximum distances within each data set did not exceed that of ITSI and ITS2 tree comparisons, the differences in the topologies of the pruned ITS1 and ITS2 trees were not significant and the hypothesis that these differences are due to sampling error can not be rejected.

Tree length increased by a distance value of seven when pruned ITS1 sequence data were made to fit the topology of the trees generated from the pruned ITS2 data set. Only a two step increase was, however, required for pruned ITS2 data to fit the topology of the trees generated from the pruned ITS1 data set.

\section{DISCUSSION}

In the present study, sequence data from the D2 region of the $28 \mathrm{~S}$ rRNA gene, the 5.8S rRNA gene and the two ITS regions of the ribosomal gene cluster were assessed for their usefulness in distinguishing among isolates of Trichoderma. These data were also assessed for their phylogenetic robustness in predicting the relations of isolates within the genus.

Sequence data from the D2 region differentiated the 50 Trichoderma isolates into groups that were mostly consistent 
(b) ITS1 MP

(a) D2 28S MP

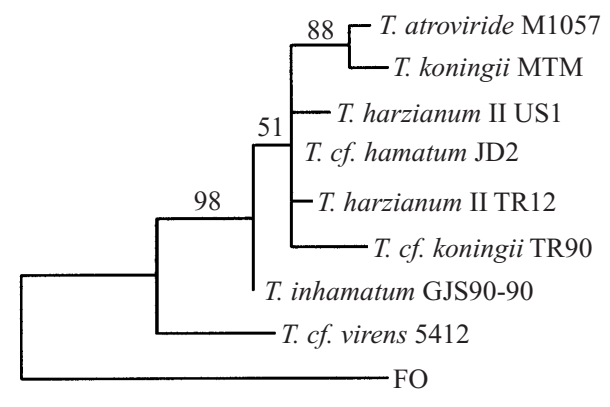

$\mathrm{CI}=0 \cdot 923$

(c) ITS2 MP

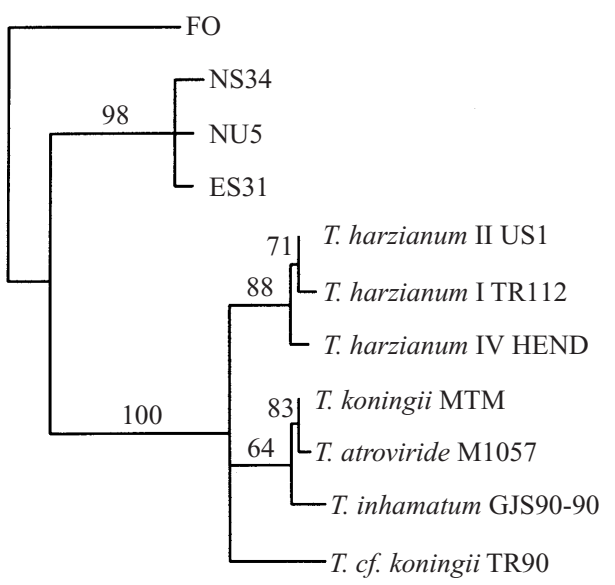

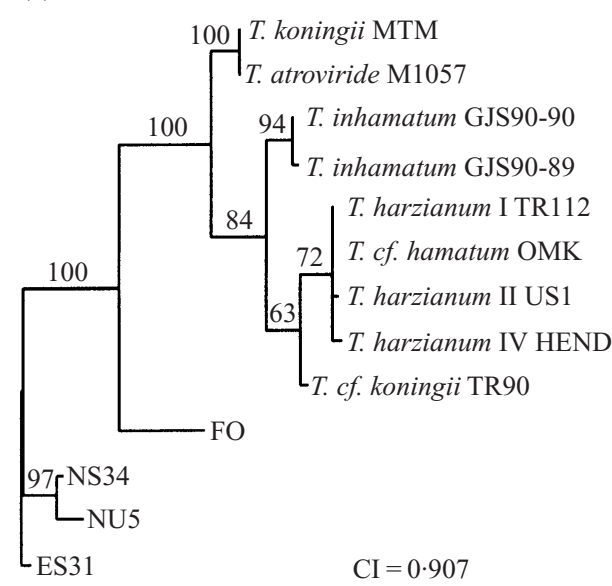

(d) ITS1-5.8S-ITS2 MP

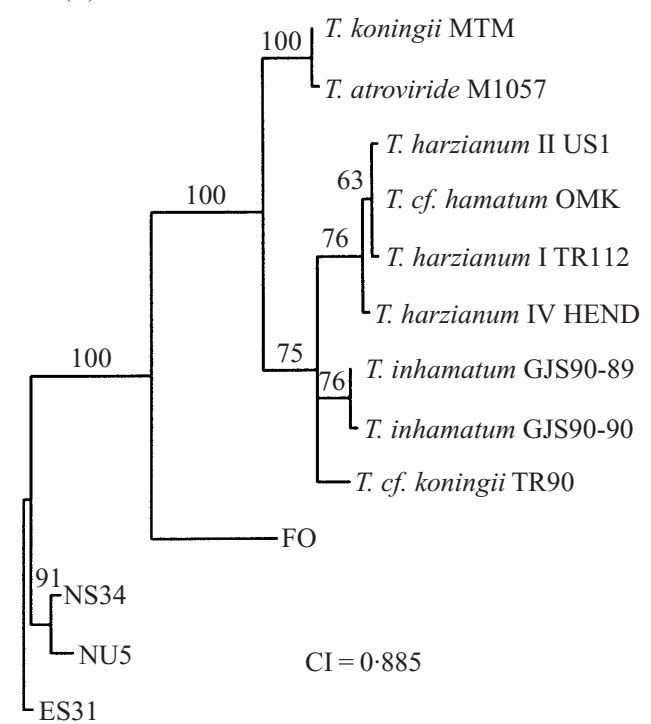

Fig. 3. Most parsimonious (MP) estimates of Trichoderma isolate phylogeny (a) for sequence data from the D2 region, with Fusarium oxysporum (FO) specified as the outgroup, and for sequence data from the (b) ITS1, (c) ITS2 and (d) ITS1, 5.8S and ITS2 combined regions, with Neotyphodium uncinatum (NU5), Neotyphodium sp. (NS34), Epichloë sp. (ES31) and F. oxysporum (FO) specified as the outgroups. Values above branches indicate the degree of bootstrap support expressed as percentage of 1000 bootstrapped trees in which the corresponding clades are present. Branches for which there are no bootstrap values occur in less than $50 \%$ of the bootstrapped trees. $\mathrm{CI}=$ consistency index.

with morphology, grouping them at a level between genus and species. These D2 groupings did not, however, correlate to the sections proposed by Bissett (1991a), since the different sub-groups of $T$. harzianum were distributed between two sequence types. The few exceptions to consistency between the D2 sequence and morphological groupings were where the $T$. atroviride isolates were separated into two different sequence Types (Types 1 and 3), the separation of six T. cf. hamatum isolates into two types (five in Type 2 and one in Type 3), and where the T. harzianum II isolates were separated into two types (seven had Type 3 sequence and one had unique sequence). Muthumeenakshi et al. (1994) and Kuhls et al. (1997) also found sequence data provided more consistent and reliable groupings of Trichoderma species than morphological identifications. Such findings emphasize the diffi- culties experienced in using morphological and cultural characters alone to differentiate isolates of this genus.

The low level of variation among isolates in the D2 sequence suggests this region was not suitable for determining phylogenetic relationships within or among species of Trichoderma. Similarly, Muthumeenakshi et al. (1994) found insufficient variability in the D2 region to distinguish among morphologically distinct isolates of the species T. harzianum.

Of the four regions of ribosomal DNA tested (D2, ITS1, $5.8 \mathrm{~S}$ and ITS2), sequence data from the ITS1 region contained the most informative data enabling the greatest differentiation among the Trichoderma isolates.

ITS sequences of our strains were compared with those from the studies of Muthumeenakshi et al. $(1994,1998)$ and Ospina-Giraldo et al. (1998) to determine whether the isolates 


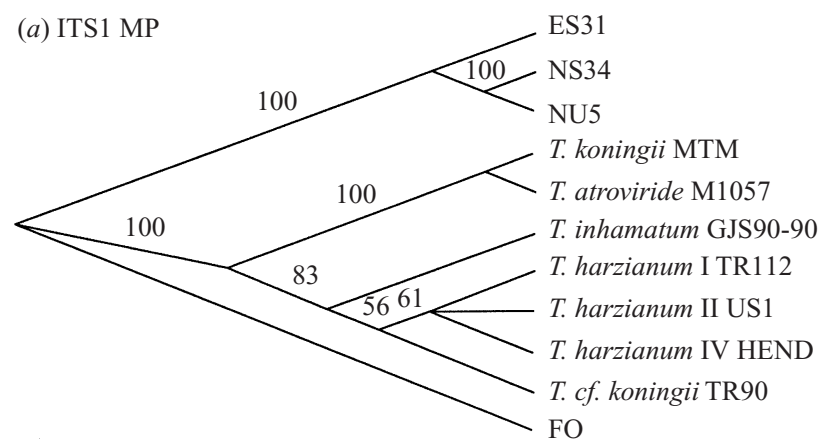

(b) ITS2 MP

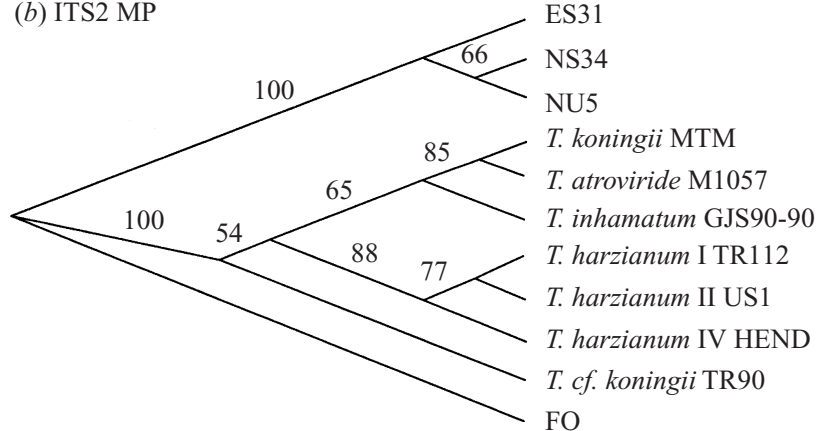

Fig. 4. Most parsimonious (MP) trees for sequence from the (a) ITS1 and (b) ITS2 data sets. The ITS1 data set has had sequence for the taxa OMK and GJS90-89 removed so that both ITS1 and ITS2 data sets have identical taxa. Both trees represent the $50 \%$ majority rule consensus tree from a bootstrapped (20 replications) heuristic search using the TBR branch swapping and random taxon addition (20 replications) options. The trees differ in their placement of the $T$. inhamatum isolate GJS90-90.

that exhibited good biological control activity were similar to those that were aggressive pathogens of commercial mushrooms in the British Isles and North America. Their studies found isolates that were aggressive colonizers of commercially grown mushrooms possessed either Th2 or Th4 type ITS1 sequence and that less aggressive isolates possessed either Th1 or Th3 type sequences. None of the strains tested in the present study possessed the aggressive type sequences, Th2 and Th4. In fact, the majority of these isolates shared greater sequence similarity to isolates of the less aggressive groups, Th1 and Th3. In particular, the two T. harzianum I isolates TR112 and TR108 shared 100\% sequence similarity to the ThI isolates.

Comparison of ITSI sequence of the T. harzianum isolates (US1, TR112, TR108, G4 and HEND) to that of the proposed 'true' T. harzianum neotype strain of Gams \& Meyer (1995) (CBS 226.95; EMBL AJ222720), revealed that US1 shared $100 \%$ nucleotide sequence similarity with it. The remaining four $T$. harzianum isolates also shared high sequence similarity to the neotype, with ThI isolates TRI12 and TR108 differing in only three nucleotide positions, and the G4 and HEND isolates differing by four. This high similarity in ITS1 sequence lends support for the identities of these isolates as 'true' $T$. harzianum. Further to this, Muthumeenakshi et al. (1998) recently proposed that their ThI isolates corresponded to the neotype T. harzianum strain, based on ITS1 sequence similarity.
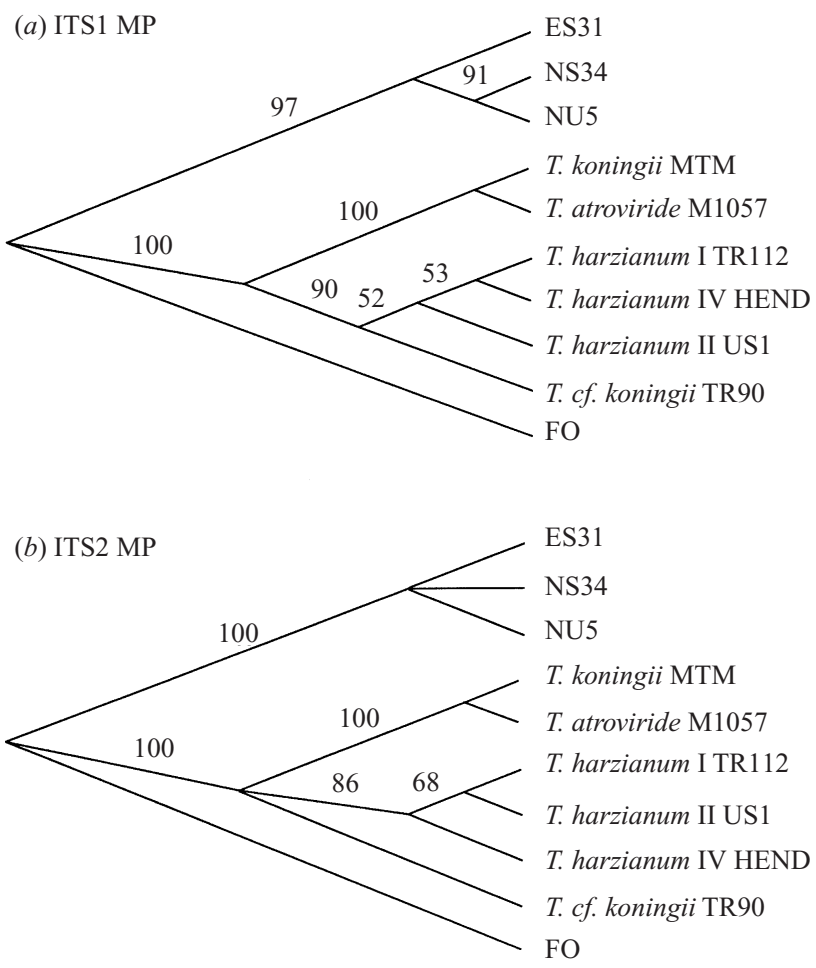

Fig. 5. Most parsimonious (MP) trees for sequence from the (a) ITS1 and (b) ITS2 data sets. In addition to the removal of OMK and GJS90-89 sequence from the ITS1 data set, both ITS1 and ITS2 data sets have had GJS90-90 sequence removed. Both trees represent the $50 \%$ majority rule consensus tree from a bootstrapped (20 replications) heuristic search using the TBR branch swapping and random taxon addition (20 replications) options.

With respect to the other species investigated, $T$. atroviride M1057, OG3, TTS, M1037, HPP1, TBHPP7, D and D74 all shared $100 \%$ ITS sequence similarity with three other $T$. atroviride isolates (GenBank X93948, X9394, Z48817), three isolates referred to as T. harzianum/atroviride (Kuhls et al., 1996) and a biocontrol T. harzianum strain LCI (Schlick et al., 1994). Of these, the T. harzianum isolates have since been reidentified as T. atroviride (W. Gams, pers. comm.). Similarly, Opsina-Giraldo et al. (1998) have reported that the $T$. harzianum Th3 group of Muthumeenakshi et al. (1994) shared high ITS sequence similarity with isolates of $T$. atroviride. Based on their results, these authors propose Th3 to be $T$. atroviride. The $T$. atroviride strains examined in our study also shared $99 \%$ sequence similarity in the ITSI region to that of the Th3 T. harzianum group of Muthumeenakshi et al. (1994). Furthermore, the morphology of our strains is consistent with that of $T$. atroviride. It is, therefore, clear that Th3 is $T$. atroviride and should henceforth be referred to as such, and not as a group of $T$. harzianum.

Similarly, T. koningii MTM differed by only one nucleotide in the ITS2 region when its ITS sequence was compared to that of the proposed T. koningii neotype (CBS 459.96, GenBank Z79628) of Liekfeldt et al. (1998).

For the remaining strains used in this study, only some of the identities were supported when ITS sequence was compared with those of the same species in the GenBank 
database. These included the T. harzianum I, II and IV isolates, TR112, TR108, HPP1, TBHPP7 and US1. In contrast, T. cf. hamatum OMK and T. inhamatum GJS90-89 and GJS90-90 shared greater sequence similarity to other species. T. cf. koningii TR90 was unique in that it not only shared $<90 \%$ ITS sequence to other T. koningii isolates in the database but also shared low sequence similarity with other Trichoderma spp., suggesting this isolate may be an outlier of Trichoderma.

With respect to the analysis of sequence data, numerous studies have been published where fungal phylogenies are predicted using ribosomal sequence data but no indication is given of how robust the data were and, therefore, how reliable the phylogenies were (for example, Bryan, Daniels \& Osbourn 1995; Glenn et al., 1996; Sreenivasaprasad et al., 1996; Kuhls et al., 1997). Results of the present study reveal the importance of conducting such tests as, in this instance, different data sets for the same group of isolates produced significantly different phylogenies. A number of steps were undertaken to test the reliability of the phylogenies produced. First, a suitable outgroup was selected. The outgroup had to be adequately removed from the Trichoderma ingroup but possess enough phylogenetic signal to place it correctly within the tree. Results show four outgroups were required to root the trees with sufficient confidence.

Secondly, for the same group of isolates, four different data sets were analysed using two different methods to determine if the data sets all produced the same phylogeny. Although the two neighbour joining and parsimony methods produced the same relative groupings of isolates for the different data sets, significant differences in topology were observed between the two ITS data sets. The order of taxa within the ITS2 sequence trees appeared to be in the reverse order to those within the ITSI trees. When the two data sets were combined and analysed, the ITS1 order dominated over the ITS2 and the resolution of some groups within the tree was poor.

The third step was to perform congruence studies on the two data sets. Results from these revealed sequence from $T$. inhamatum GJS90-90 and GJS90-89 was corrupting tree topologies. The T. inhamatum isolates differed in ITS sequence by just one nucleotide in the ITSI region. Waalwijk et al. (1996) observed a similar discordance between ITS1 and ITS2 sequence based phylogenies generated for species of Fusarium and suggest that the differences may be due to the hypothetical co-existence of several ITS types in the ancestral Fusarium species. The recent discovery of more than one sequence type in the ITS2 region of individual Gibberella fujikuroi isolates lends support to this (O'Donnell, Cigelnik \& Nirenberg, 1998).

For our study, however, an alternative explanation might be that the sequence of T. inhamatum was corrupting the ITS trees and may be doing so because it could potentially be a hybrid of the two different groups to which it was assigned. By referring to sequence data and comparing the number of nucleotides that GJS90-90 had in common with each group (i.e. the MTM and M1057 group and the TR112, US1 and HEND group), it appears that GJS90-90 shares sequence with the TR112, USI and HEND group in the ITSI, the 5.8S regions plus the first 76 nucleotides of the ITS2 region. For the remainder of the ITS2 region and the D2 region of the $28 \mathrm{~S}$ like
rRNA gene, which lies downstream from the ITS2 region, there was an almost equal number of shared nucleotides with each group. This overall pattern suggests that a recombination event could have occurred within the ITS2 region of this isolate. In which case, the ITS1 and initial ITS2 regions represent one end of the recombination event and the rest of the ITS2 and the D2 regions represent a region of heteroduplex DNA.

In further support of this explanation, the $700 \mathrm{bp}$ stretch of proposed heteroduplex DNA falls within the average length (i.e. $1.5 \mathrm{~kb}$ long) of heteroduplex regions observed in yeast (Borts \& Harber, 1987). Results of the present study, therefore, suggest that the tree congruence studies of Rodrigo et al. (1993) may provide an alternative method for determining the presence of recombinant DNA and the sites of recombination events in fungal genomes as opposed to the more traditional restriction site polymorphism studies.

By removing $T$. inhamatum sequence from both data sets, it was found that both data sets essentially produced the same topologies. Differences can be attributed to the lack of phylogenetic information within the ITS2 region as indicated by the fact that ITS2 sequence data was close to producing the ITSI tree topology (i.e. an increase in tree length of only two when ITS2 data was made to fit the ITS1 tree topology). Had any of the original phylogenies been taken at face value, some of the isolate relationships would have been in error, based on an incorrect phylogeny.

Examination of the pruned phylogenetic ITS trees show morphological species were not always clearly defined. This is illustrated by the fact that the species $T$. koningii had strains 'misplaced' in the same clade as isolates identified as a different morphological species. In other words T. koningii was paraphyletic and its phylogenetic relationship to other species within the genus was unresolved. Kuhls et al. (1997) also found morphological species of Trichoderma, including $T$. koningii, to be paraphyletic when the ITS sequence was analysed using the maximum parsimony method. Similarly, analysis of other molecular and biochemical data also support this finding. For example, Stasz et al. (1989) found morphological species of Trichoderma to be paraphyletic when isoenzyme data from 71 isolates, representing four morphological species, were analysed using the parsimony method. Leuchtmann, Petrini \& Samuels (1996) also found UPGMA analysis of isoenzyme data identified paraphyletic Trichoderma species.

Although not always consistent with morphology, the grouping of Trichoderma isolates based on ITS and D2 sequences was conserved between the two data sets. For instance, isolates sharing a common D2 sequence (TR112, HEND and USI) were also grouped together in both ITS trees. In addition, the close relationship between T. atroviride M1057 and T. koningii MTM was also conserved. Although the ITSI sequence gave the best overall estimate of the phylogenetic tree, like the other sequence data, this data could not be used to resolve phylogenetic relationships among isolates sharing the Type 3 D2 sequence. More data would be required to fully resolve these phylogenetic relationships. Analysis of other variable regions within the genome may provide the required level of variability. 
We are grateful to Jo Bowen, Matt Templeton and Eric Rikkerink for their valuable advice and assistance. Thanks also to Walter Gams and Wieland Meyer for supplying us with the ITS sequence of their T. harzianum neotype strain. This work was funded by the New Zealand Foundation for Research and Technology, contract number CO6517.

\section{REFERENCES}

Altschul, S. F., Gish, W., Miller, W., Myers, E. W. \& Lipman, D. J. (1990). Basic local alignment search tool. Journal of Molecular Biology 215, 403-410.

Bissett, J. (1984). A revision of the genus Trichoderma. I. Section Longibrachiatum sect. nov. Canadian Journal of Botany 62, 924-931.

Bissett, J. (1991a). A revision of the genus Trichoderma. II. Infrageneric classification. Canadian Journal of Botany 69, 2357-2372.

Bissett, J. (1991 b). A revision of the genus Trichoderma. III. Section Pachybasium. Canadian Journal of Botany 69, 2373-2417.

Bissett, J. (1991 c). A revision of the genus Trichoderma. IV. Additional notes on section Longibrachiatum. Canadian Journal of Botany 69, 2418-2420.

Bissett, J. (1992). Trichoderma atroviride. Canadian Journal of Botany 70, 639-641.

Borts, R. H. \& Harber, J. E. (1987). Meiotic recombination in yeast: Alteration by multiple heterozygosities. Science 237, 1459-1465.

Bryan, G. T., Daniels, M. J. \& Osbourn, A. E. (1995). Comparison of fungi within the Gaeumannomyces-Phialophora complex by analysis of ribosomal DNA sequences. Applied and Environmental Microbiology 61, 681-689.

Buchert, J., Ranua, M., Kantelinen, A. \& Viikari, L. (1992). The role of two Trichoderma reesei xylanases in the bleaching of pine kraft pulp. Applied Microbiology and Biotechnology 37, 825-829.

Crowhurst, R. N., Hawthorne, B. T., Rikkerink, E. H. A. \& Templeton, M. D. (1991). Differentiation of Fusarium solani f.sp. cucurbitae races 1 and 2 by random amplification of polymorphic DNA. Current Genetics 20, 391-396.

Felsenstein, J. (1985). Confidence limits in phylogenies: An approach using the bootstrap. Evolution 39, 783-791.

Gams, W. \& Meyer, W. (1995). Delimitation, typification and nomenclature of Trichoderma harzianum. In Proceedings of the Fifth International Trichoderma/Gliocladium Workshop, p. 12. Beltsville, MD, U.S.A., 18-20 April.

Ghisalberti, E. L. \& Sivasithamparam, K. (1991). Antifungal antibiotics produced by Trichoderma spp. Soil Biology and Biochemistry 11, 1011-1020.

Glenn, A. E., Bacon, C. W., Price, R. \& Hanlin, R. T. (1996). Molecular phylogeny of Acremonium and its taxonomic implications. Mycologia $\mathbf{8 8}$, 369-383.

Guadet, J., Julien, J., Lafay, J. F. \& Brygoo, Y. (1989). Phylogeny of some Fusarium species, as determined by large-subunit rRNA sequence comparison. Molecular Biology and Evolution 6, 227-242.

Hillis, D. M., Allard, M. W. \& Miyamoto, M. M. (1993). Analysis of DNA sequence data: Phylogenetic inference. Methods in Enzymology 224, 456-486.

Jukes, H. J. \& Cantor, C. R. (1969). Evolution of protein molecules. In Mammalian Protein Metabolism, Vol. 3 (ed. H. N. Munro), pp. 21-132. Academic Press: New York, U.S.A.

Kimura, M. (1980). A simple method for estimating evolutionary rates of base substitutions through comparative studies of nucleotide sequences. Journal of Molecular Evolution 16, 111-120.

Kluge, A. G. \& Farris, J. S. (1969). Quantitative phyletics and the evolution of anurans. Systematic Zoology 18, 1-32.

Kuhls, K., Lieckfeldt, E., Samuels, G. J., Kovacs, W., Meyer, W., Petrini, O., Gams, W., Börner, T. \& Kubicek, C. P. (1996). Molecular evidence that the asexual industrial fungus Trichoderma reesei is a clonal derivative of the ascomycete Hypocrea jecorina. Proceedings of the National Academy of Sciences U.S.A. 93, 7755-7760.

Kuhls, K., Lieckfeldt, E., Samuels, G. J., Meyer, W., Kubicek, C. P. \& Börner, T. (1997). Revision of Trichoderma sect. Longibrachiatum including related teleomorphs based on analysis of ribosomal DNA internal transcribed spacer sequences. Mycologia 89, 442-460.

Kumar, S., Tamura, K. \& Nei, M. (1993). MEGA: Molecular Evolutionary
Genetics Analysis, Version 1.01. The Pennsylvania State University University Park, PA 16802, U.S.A.

Leuchtmann, A., Petrini, O. \& Samuels, G. J. (1996). Isozyme subgroups in Trichoderma section Longibrachiatum. Mycologia 88, 384-394.

Lieckfeldt, E., Samuels, G. J., Börner, T. \& Gams, W. (1998). Trichoderma koningii: Neotypification and Hypocrea teleomorph. Canadian Journal of Botany 76, 1507-1522.

Loeppke, C. B., Ronald, M. D., Sprouse, R. F., Carlson, J. V. \& Everett, E. D. (1983). Trichoderma viride peritonitis. Southern Medical Journal 76, 798-799. Margush, T. \& McMorris, F. R. (1981). Consensus n-trees. Bulletin of Mathematical Biology 43, 239-244.

Menzies, J. G. (1993). A strain of Trichoderma viride pathogenic to germinating seedlings of cucumber, pepper and tomato. Plant Pathology 42, 784-791.

Muthumeenakshi, S., Brown, A. E. \& Mills, P. R. (1998). Genetic comparison of the aggressive weed mould strains of Trichoderma harzianum from mushroom compost in North America and the British Isles. Mycological Research 102, 385-390.

Muthumeenakshi, S., Mills, P. R., Brown, A. E. \& Seaby, D. A. (1994) Intraspecific molecular variation among Trichoderma harzianum isolates colonizing mushroom compost in the British Isles. Microbiology 140 769-777.

O’Donnell, K., Cigelnik, E., Nirenberg, H. I. (1998). Molecular systematics and phylogeography of the Gibberella fujikuroi species complex. Mycologia 90 , 465-493.

Ospina-Giraldo, M. D., Royse, D. J., Thon, M. R., Chen, X. \& Romaine, C. P. (1998). Phylogenetic relationships of Trichoderma harzianum causing mushroom green mold in Europe and North America to other species of Trichoderma from world-wide sources. Mycologia 90, 76-81.

Papavizas, G. C. (1985). Trichoderma and Gliocladium: Biology, ecology, and potential for biocontrol. Annual Review of Phytopathology 123, 23-54.

Rehner, S. A. \& Samuels, G. J. (1994). Taxonomy and phylogeny of Gliocladium analysed by large subunit rDNA sequences. Mycological Research 98, 625-634.

Rifai, M. A. (1969). A revision of the Genus Trichoderma. Mycological Papers 116, 1-56.

Rodrigo, A. G., Kelly-Borges, M., Bergquist, P. R. \& Bergquist, P. L. (1993). A randomisation test of the null hypothesis that two cladograms are sample estimates of a parametric phylogenetic tree. New Zealand Journal of Botany 31, 257-268.

Saitou, N. \& Nei, M. (1987). The neighbour-joining method: A new method for reconstructing phylogenetic trees. Molecular Biology and Evolution 4, 406-425.

Samuels, G. J. (1996). Trichoderma: a review of biology and systematics of the genus. Mycological Research 100, 923-935.

Samuels, G. J., Lieckfeldt, E. \& Nirenberg, H. I. (1999). Trichoderma asperellum, a new species with warted conidia, and redescription of T. viride. Sydowia 51, 71-88.

Schirmböck, M., Lorito, M., Wang, Y., Hayes, C. K., Arisan-Atac, I., Scala, F., Harman, G. E. \& Kubicek, C. P. (1994). Parallel formation and synergism of hydrolytic enzymes and peptaibol antibiotics, molecular mechanisms involved in the antagonistic action of Trichoderma harzianum against phytopathogenic fungi. Applied and Environmental Microbiology 60 4364-4370.

Schlick, A., Kuhls, K., Meyer, W., Lieckfeldt, E., Börner, T. \& Messner, K. (1994). Fingerprinting reveals gamma-ray induced mutations in fungal DNA: implications for identification of patent strains of Trichoderma harzianum. Current Genetics 26, 74-78.

Seaby, D. (1987). Infection of mushroom compost by Trichoderma species. Mushroom Journal 179, 355-361.

Sreenivasaprasad, S., Mills, P. R., Meehan, B. M. \& Brown, A. E. (1996). Phylogeny and systematics of 18 Colletotrichum species based on ribosomal DNA spacer sequences. Genome 39, 499-512.

Stasz, T. E., Nixon, K., Harman, G. E., Weeden, N. F. \& Kutler, G. A. (1989). Evaluation of phenetic species and phylogenetic relationships in the genus Trichoderma by cladistic analysis of isozyme polymorphism. Mycologia $\mathbf{8 1}$ 391-403.

Swofford, D. L. (1993). PAUP: Phylogenetic Analysis Using Parsimony, Version 3.1.1. Illinois Natural History Survey: Champaign, U.S.A.

Waalwijk, C., de Koning, J. R. A., Baayen, R. P. \& Gams, W. (1996). 
Discordant groupings of Fusarium spp. from sections Elegans, Liseola and Dlaminia based on ribosomal ITS1 and ITS2 sequences. Mycologia 88 361-368.

White, T. J., Bruns, T., Lee, S. \& Taylor, J. (1990). Amplification and direct sequencing of fungal ribosomal RNA genes for phylogenetics. In $P C R$ Protocols. A Guide to Methods and Applications (ed. M. A. Innis, D. H. Gelfand, J. J. Sninsky \& T. J. White), pp. 315-322. Academic Press: San Diego, U.S.A.

(Accepted 12 April 1999) 